\title{
Targeting the mercapturic acid pathway and vicenin-2 for prevention of prostate cancer
}

Sharad S Singhal ${ }^{1}$, Divya Jain ${ }^{2}$, Preeti Singhal ${ }^{3}$, Sanjay Awasthi ${ }^{4}$, Jyotsana Singhal ${ }^{1}$, and David Horne ${ }^{1}$

${ }^{1}$ Department of Molecular Medicine, Beckman Research Institute of City of Hope, Comprehensive Cancer Center and National Medical Center, Duarte, CA 91010; ${ }^{2}$ Department of Ophthalmology, University College of Medical Sciences and Guru Teg Bahadur Hospital, New Delhi 110095, India; ${ }^{3}$ University of Texas Health, San Antonio, TX 78229; ${ }^{4}$ Texas Tech University Health Sciences Center, Lubbock, TX 79430

\section{Running Title: $\quad$ Prostate Cancer Chemoprevention by VCN-2 \\ Journal Category: Review Article}

Address correspondence to: $\quad$ Sharad S Singhal, Ph.D., Professor, Phone: 626-218-4238; Fax: 626301-8136; Email: ssinghal@coh.org

The abbreviations used are: AICaP, androgen-independent prostate cancer; AR, androgen receptor; ASCaP, androgen-sensitive prostate cancer; $\mathrm{CaP}$, carcinoma of prostate; $\mathrm{CRCaP}$, castration resistant carcinoma of prostate; CD31, cluster of differentiation 31 protein; cdc2, cyclin-dependent kinase 1 also known as CDK1 or cell division cycle protein 2 homolog; CDE, clathrin-dependent endocytosis; CDK4, cyclin dependent kinase 4; DTL, docetaxel; EGFR, epidermal growth factor receptor; GSH, glutathione; GS-E, glutathione electrophile conjugates; GST, glutathione S-transferase; 4HNE, 4-hydroxy nonenal; Hsf1, heat shock transcription factor 1; hTERT, human telomerase reverse transcriptase; IGF-1R, insulin growth factor 1 receptor; LOOH, lipid-hydroperoxides; MAP, mercapturic acid pathway; mTOR, mammalian target of rapamycin; OSL, Ocimum Sanctum Linn; PCNA, proliferative cell nuclear antigen; PIN, prostatic intraepithelial neoplasia; PSA, prostate specific antigen; PSMA, prostate specific membrane antigen; PTEN, phosphatase and tensin homologue; RB, retinoblastoma protein; RLIP76 (RALBP1), a 76 kDa Ral-interacting protein; VCN-2, vicenin-2; VEGF, vascular endothelial growth factor 


\begin{abstract}
Prostate cancer (CaP) is often androgen-sensitive malignancy and regresses upon inhibition of androgen signaling. However, CaP, nearly always develops androgen resistance and progresses to aggressive and lethal androgen-independent CaP, which lacks satisfactory therapy. For metastatic CaP, patients are often treated with Taxotere (docetaxel), a cytoskeleton-targeted chemotherapy drug, that provides transient palliative benefit but to which patients rapidly develop drug-resistance. Combination chemotherapy may be used instead, but is more toxic and adds little clinically relevant benefit over docetaxel. Therefore, novel strategies to enhance docetaxel efficacy are needed to effectively treat patients with metastatic CaP. The mercapturic acid pathway, which metabolizes genotoxic and pro-apoptotic toxins, is over-expressed in CaP and plays an important role in carcinogenesis, metastasis and therapy-resistance of CaP. Vicenin2, a flavonoid derived from Tulsi (holy basil) as an active compound, inhibits the growth of CaP and increases the anti-tumor activity of docetaxel in-vitro and in-vivo. Taken together, the combination of vicenin-2 and docetaxel could be highly effective in the treatment of advanced and metastatic CaP due to their multi-targeting anti-tumor potential.
\end{abstract}

Key Words: Prostate cancer; vicenin; RLIP76 (RalBP1); drug-resistance; glutathione-conjugate transport; clathrin-dependent endocytosis 


\section{Introduction}

Prostate cancer (CaP, carcinoma of prostate) constitutes a major health problem in Western countries. $\mathrm{CaP}$ is a type of malignancy that arises in the prostate gland, tends to develop in older men, aged 50 and over, and in many cases CaP develops slowly, although in some cases it is aggressive and metastasizes to other parts of the body [1-3]. Dietary factors, lifestyle-related factors, and androgens (AR) contribute to the risk of developing $\mathrm{CaP}$. Hypermethylation of $\mathrm{CpG}$ island sequences encompassing the regulatory region of glutathione S -transferase (GSTP1) may link exposure to genome-damaging stress to increased genomic instability during prostatic carcinogenesis. GSTs are detoxifying enzymes that catalyze conjunction of glutathione (GSH) with harmful, electrophilic molecules, thereby protecting cells from carcinogenic agents. The identification of key molecular alterations in CaP cells has implicated carcinogen defenses, such as GSTP1, growth-factor-signaling pathways (i.e., NKX3.1, PTEN, and p27), and AR as critical determinants of the phenotype of CaP cells. In addition, this has defined specific targets that can be used to detect, diagnose, and treat CaP [3-5]. For example, in prostatic intraepithelial neoplasia (PIN), cells that lack GSTP1, undergo genomic damage mediated by carcinogens. NKX3.1, PTEN, and p27 regulate the growth and survival of prostate cells in the normal prostate. NKX3.1 binds DNA and represses expression of the prostate-specific antigen (PSA) gene. The gene for phosphatase and tensin homologue (PTEN), a tumor-suppressor gene, is a common target for somatic alteration during the progression of CaP. PTEN is expressed in normal epithelial cells and in PIN. However, in CaP, levels of PTEN are frequently reduced, particularly in cancers of a high grade or stage [4,5]. Reduced levels of PTEN and NKX3.1 lead to reduced levels of p27, a cyclin-dependent kinase inhibitor encoded by the CDKN1B gene, which contributes to increased proliferation and decreased apoptosis of CaP cells and thus to poor prognosis. In this way, haploinsufficiency for PTEN and NKX3.1 may promote abnormal proliferation of prostate cells [3,4].

Treatment of CaP usually involves therapeutic reduction in the levels of testosterone and dihydrotestosterone. However, this is complicated by the eventual emergence of androgen-independent prostate cancer (AICaP), which is associated with mutations in the androgen-receptor that permit receptor activation by other ligands, increased expression of androgen-receptors accompanying AR amplification, and ligand-independent androgen-receptor activation [5-8]. During androgen withdrawal therapy, the AR signal transduction pathway can also be activated by amplification of the AR gene, by AR gene mutations, or by altered activity of AR co-activators. All of these mechanisms contribute to the emergence of AICaP [6-8]. 
Furthermore, CaP is molecularly complex, having a multifocal and heterogeneous nature, which presents challenges to interventions that act on a single target [9-12]. The fact that 1 in 3 men develop PIN and that 1 in 6 men develop CaP reflects the associated life-time risk for CaP [9]. In addition, given the dynamic molecular plasticity of incident and progressing $\mathrm{CaP}$ tumors, rapid emergence of drugresistance, and dose-limiting toxicities of currently used drugs like docetaxel (DTL) [13, 14], there is high interest in identifying novel, safe, and non-toxic natural anticancer compounds that can inhibit development of $\mathrm{CaP}$ and/or synergistically enhance its sensitivity to anticancer drugs. A variety of natural herbal compounds that have inherent chemical antioxidant properties offer a potential means to overcome these challenges because they display multi-targeted anticancer activities [15-17]. Therefore it may be beneficial to exploit these herbs for human therapy, but challenges remain in identifying appropriate agents or compounds for study.

Phenolic antioxidant compounds that have apoptotic effects on cancer cells appear to work through pleiotropic mechanisms: that simultaneously inhibits multiple pro-cancer pathways [18, 19]. How this happens remains unclear? Assuming directional signaling (up-stream to down-stream), one must consider at least four possible explanations: 1) the drug acts on one target, and all of the observed effects (i.e., inhibition of Akt, MEK, p53, AR, cMyc, etc.) are down-stream of that target; 2) the compound acts on many targets and each effects arises from direct inhibition of each target by the compound; 3) the compound is does not directly act on any of the targets, but changes the cellular milieu such that the up- and down-stream targets are simultaneously affected; or 4) a combination of 1, 2 and 3. The first explanation is unlikely because of the known ability of phenolic antioxidants to directly bind many different proteins. The second is also unlikely because, even if the compound binds all targets, the structural differences in the target proteins make it highly unlikely that a single molecule (or even its metabolites) could inhibit the activity of all targets. Given that antioxidants, by nature, reduce overall concentrations of oxidizing species, a change in milieu appears much more likely. However, given the demonstrated ability of some phenolic compounds to bind to and specifically inhibit one or more signaling proteins, and that inhibition of an upstream signaling protein will necessarily reduce signaling to the next, explanation 4 (a combination of 1-3) is the most likely scenario. In addition, the third scenario should predominate because each of the targets under consideration here is known to be directly affected by overall oxido-reductive balance. Indeed, the in-vitro measures of the catalytic or binding activities of all of the signaling proteins being considered are directly regulated by the ratio of reduced $v$ s. oxidized thiols (i.e., a requirement for $\beta$-mercaptoethanol in the buffers used to measure their activities). The ratio of the oxidized:reduced cellular thiols is inextricably linked and in equilibrium with all redox pairs. Thus, 
the chemical antioxidant effect of any such compound would alter the general milieu such that the activities of all potential targets in a cascade should be affected. Because oxidative stress can activate many of these signaling proteins (i.e., Akt, p53, сMyc, MEK), it seems reasonable that reducing this stress by using an antioxidant should inhibit their activation. However, activation of apoptosis through the intrinsic Bcl-2/Bax mechanism generally happens in concert with an oxidative stress [20-22]. Thus, none of these explanations are entirely satisfactory, and an alternative model must be considered.

The cancer-preventive actions of polyphenolic phytochemicals were originally defined by studying their effects on the mercapturic acid pathway (MAP). Specifically, compounds that induced GST (phase-II detoxification and rate limiting enzyme of MAP) and suppressed cytochrome p450 (phase I detoxification enzymes, upstream of GSTs in the MAP) were identified as being antioxidant cancerpreventives and those that exerted the opposite effect were pro-oxidants. Because all antioxidants are also pro-oxidants at higher concentrations, it stands to reason that those compounds that could prevent cancer by reducing oxidative stress at low concentrations could at high concentrations exert oxidative stress that might trigger apoptosis in cancer cells [23-25]. In this regard, it is interesting that polyphenols exert antioxidant effects in the nanomolar concentration range (which induces GSTs), but apoptosis of cancer cells requires micromolar concentrations (which suppresses or inhibits GSTs). Although this model is conceptually reasonable, it does not explain why increased oxidative stress (due to very high concentrations of polyphenols) could inhibit the activation of p53, cMyc, or Akt.

We argue that the MAP-Ral hypothesis could explain some of these paradoxical findings and link the chemical/biochemical effects of phenolic antioxidants with well-understood kinase signaling pathways. In this model, high concentrations of polyphenols inhibit the MAP at GST, resulting in further exacerbation of oxidative stress. Compounds that block the glutathione-electrophile-conjugate (GS-E) transporter, RLIP76, could inhibit the Ras/Raf/MEK/Myc or AKT/mTOR/MDM/p53 pathways by simultaneously inhibiting endocytosis [26, 27]. The resultant loss of stress-resistance and cell-cycling checkpoint combined with increased oxidative stress due to the chemical effect of high polyphenol concentrations could shift the balance between Bcl2 and Bax towards apoptosis [20-22]. In recent years, the importance of the Ral/MEK/Myc pathway in cancer has been recognized [28-30]. Blockade of the Ral effector RLIP76 could also exacerbate the tendency towards apoptosis [27-32]. Because Ral is a primary regulator of exosomes, a tendency towards apoptosis would be further compounded if exosomes function to potentiate ligand-receptor signaling. 
Flavonoids can modulate drug action through multiple mechanisms such as influencing intestinal absorption, altering the rate and nature of metabolism, bio-distribution of drugs and, most importantly, affecting parallel signaling networks in targeted cancers when co-administered with chemotherapy drugs resulting in additive, antagonistic or synergistic effects [16-19]. We initially revealed a synergistic effect between the flavonoid VCN-2 and docetaxel (DTL), and in in-vivo studies, showed that orally administered VCN-2 in combination with DTL was effective in AICaP [33].

Androgen-independent metastatic prostate cancer (AICaP) is a highly prevalent, morbid, debilitating and lethal disease of men for which satisfactory prevention and treatment are lacking [10]. The use of DTL in advanced CaP provides transient palliative benefit, followed by resistance [13], which necessitates combinatorial tertiary chemopreventive strategies. Novel pharmacological methods for primary prevention of $\mathrm{CaP}$ and to overcome tumor burden of metastatic disease are urgently needed. We have sought to decipher the role of the MAP enzymes in carcinogenesis, metastasis, and drug-resistance, and have shown that this pathway plays a key role in apoptosis caused by diverse stressors ranging from radiation and chemotherapy, to hormone-withdrawal [28, 31-36]. Recent studies have directly linked the MAP enzymes to carcinogenesis, survival-promoting, and proliferation-promoting pathways, including cMyc, p53, Rb, and Akt, in a variety of cancers in which MAP enzymes are highly enriched, including CaP [28, 31-33, 37]. Based on this rationale, we performed in-silico docking studies with MAP enzymes to screen a group of plant-derived antioxidants, and identified a lead candidate, VCN-2. We examined VNC-2 effects in cell lines and human xenograft models of CaP and found that VCN-2 exerts strong antineoplastic activity alone and in synergy with DTL in both CaP and AICaP [33]. In this regard, herein we describe the anticancer properties of the Tulsi-derived novel flavonoid VCN-2 as a potential inhibitor of CaP growth because of its strong predicted interactions with multiple enzymes and regulatory nodes in signaling pathways that regulate MAP. The novel features of this compound include its oral bioavailability, lack of toxicity, and mechanistic relevance to central signaling proteins implicated in CaP.

Vicenin-2 (VCN-2) VCN-2 is a commercially available flavonoid (Quality Phytochemicals LLC., Edison, NJ) that is enriched in Tulsi (also known as holy basil, Ocimum sanctum Linn, OSL; family: Lamiaceae), an extensively cultivated Indian herb that has high religious significance and is believed to prolong the life of men (Fig. 1). OSL is known for its anti-inflammatory, anticancer and anti-diabetic properties in Ayurveda, and has a safe record of human consumption as a raw paste or as an herbal tea for millennia [38-45]. Oxidative stress is a well established cause in the initiation and progression of cancers [46]. Anti-oxidant and anti-inflammatory compounds like cyclooxygenase-2 inhibitors, natural 
compounds like silibinin and other flavonoids are currently being investigated as potential CaP treatments [47-49]. OSL has been investigated for its chemopreventive properties in dimethyl-benzanthracene (DMBA)-induced oral cancer [50], and also functions as a radio-protectant for bone marrow cells [51-53]. Overall, VCN-2 targets multiple nodes of $\mathrm{CaP}$ pathogenesis and progression, which also exhibiting a potent synergistic effect with orally given DTL. This provides a strong rationale for further studying VCN-2 as a single agent for the prevention of CaP and the combination of VCN-2 and DTL for targeting AICaP [33]. Further supporting this, other key features of VCN-2 are: a) hepatoprotective activity, b) antioxidant and anti-inflammatory activities, c) could act as a UV light barrier to protect the plants, d) anticancer activity, and DTL co-administration is more effective than either of the single agents in AlCaP, and e) may be a useful lead for developing multiple target-oriented therapeutic modalities for the treatment of diabetes and diabetes-associated complications [39, 41-45, 53].

Docetaxel (DTL) Currently, DTL is the drug of choice for treating AICaP and is administered intravenously (i.v.) at a dose of $60-100 \mathrm{mg} / \mathrm{m}^{2}\left(75 \mathrm{mg} / \mathrm{m}^{2}\right.$ is the recommended starting dose for AICaP) once every three weeks in clinical practice. However, i.v. administration of DTL is associated with doselimiting toxicities such as febrile neutropenia and associated myelotoxicity, which has lead to characterization of alternate routes of administration and potential combinatorial regimens. Even anemia and non-febrile neutropenia, which occur in approximately $41 \%$ and $67 \%$ of patients given DTL, can severely affect the quality of life and consequent survival in elderly patients, especially given that many $\mathrm{CaP}$ patients have extensive comorbidities (e.g., diabetes mellitus and age related decline in immune function). Myelotoxicity or bone marrow toxicity results in direct, high level of exposure to bolus doses of DTL, as is typical with i.v. administration. Although, some of the previous studies focused on trying to switch from three week to weekly doses involving moderate dosage reduction, substantial adverse effects still occurred, including hyperlacrimation, and skin- and nail-toxicity [13]. In this regard, VCN-2 in combination with DTL synergistically inhibited the growth of prostate tumors in-vivo with a greater decrease in the levels of AR, pIGF1R, pAkt, PCNA, cyclin D1, Ki67, CD31, and increase in E-cadherin [33]. The salient feature of this study was the potent synergistic effect of VCN-2 and low dose DTL (0.03 $\mathrm{mg} / \mathrm{m}^{2} \mathrm{DTL}$ and $3 \mathrm{mg} / \mathrm{m}^{2} \mathrm{VCN}-2$ given orally on alternate days as compared to the clinical dose in metastatic AICaP - $75 \mathrm{mg} / \mathrm{m}^{2}$ of DTL i.v. once every 3 weeks plus $5 \mathrm{mg}$ prednisone taken twice daily), which strongly supports further development of VCN-2 and DTL combinatorial regimens for clinical use in aggressive AICaP. 
Advancing the field of CaP research A major barrier to improving the effectiveness of treatment for AICaP is an incomplete understanding of why AICaP is so aggressive and resistant to therapy. The role of Ral, the MAP, and exosome secretion in resistance to AICaP treatment is unclear. Although, these pathways are known to be linked to therapy resistance in CaP, the underlying mechanism was not known. The recent discovery thatVCN-2 can simultaneously exploit these mechanisms to improve the effectiveness of DTL provides a paradigm shifting approach for understanding how new treatments of AICaP can be devised. This concept is supported by a wide array of literature and studies from our [28, 31-37, 54-58] and others [59-62] laboratories on the role of the MAP in the formation, growth, and metastasis of CaP. The novelty is based on a broadly-supported scientific basis: a) Ral-binding protein (RALBP1/RLIP76) functions at the intersection of the Ral and MAP pathways, b) Blockade of RLIP76 exerts potent anticancer effects on CaP, c) RLIP76, a cancer survival-promoting enzyme, is a major constituent of the exosomes secreted by $\mathrm{CaP}, \mathbf{d}$ ) a novel mechanism for the association of exosomes with the aggressive behavior of AICaP, e) identification of VCN-2 as an anticancer compound, and $\mathbf{f}$ ) the ability of VCN-2 to potentiate DTL though the MAP, a pathway not affected by DTL. This suggests that the VCN-2 and DLT should have non-overlapping toxicities, the holy grail of combination chemotherapies. Thus, the proposed characterization of the detailed molecular and pharmacokinetic mechanisms of action would lay strong scientific foundation for further clinical development of VCN-2 and DTL regimen for the effective management of human CaP (Fig. 2).

Ultimate applicability The proposed strategy would benefit patients with metastatic AICaP by improving the effectiveness of DTL chemotherapy. VCN-2 could be given orally during DTL chemotherapy of AICaP. BecauseVCN-2 blocks the androgen receptor; it could be used in the treatment of androgen-sensitive CaP (ASCaP) to avoid castration (surgical or chemical), a therapeutic approach with numerous highly undesirable side effects. If shown in further studies to prevent CaP development, VCN-2 could also be an effective preventative agent. The ability of VCN-2 to affect the composition of exosomes could be an exciting finding. The oral bioavailability of VCN-2 is particularly attractive for its pharmacological development. In addition, VCN-2 qualifies as a 'generally regarded as safe' substance, with no known toxicities at present. Our studies [33] will advance scientific knowledge on the role of Ralsignaling in CaP biology, identify an entirely novel mechanism of action of antioxidant antineoplastic agents, and allow development of new strategies to overcome DTL-resistance in AICaP. Overall, VCN-2 caused apoptosis and enhances DTL activity by blocking signaling down-stream of EGF and IGF by inhibiting MAP enzymes that regulate Ral-mediated exosome-secretion and cytoskeletal remodeling. 

(phase II biotransformation) that disposes of exogenous and endogenous toxins [23-25, 28]. It defends against exogenous electrophilic (electron-deficient) compounds that cause mutations by forming adduct with DNA-bases. The MAP is also responsible for metabolism of both DTL and VCN-2. DTL is mainly metabolized by the p450 isoenzymes CYP3A4 and CYP3A5 into well-characterized inactive hydroxyl and hydroxyoxazolidinone metabolites. Because polyphenols inhibit the metabolism of taxanes by p450 enzymes [63], it is quite possible that potentiation of DTL could be due to inhibition of its metabolism. This possibility could be tested by measuring concentrations of un-metabolized DTL (active constituent) in cells without of with VCN-2 treatment to determine whether the parent drug concentration is increased upon exposure to VCN-2. These studies are critical to elucidating the mechanism of synergy.

In contrast to DTL, nothing specific is known about VCN-2 metabolism. In general, polyphenols can also be metabolized by p450 into electrophilic derivatives that are then conjugated with GSH. It is possible that VCN-2 also has a similar fate. This is relevant because GS-Es are potent product-inhibitors of GSTs, and their formation could significantly reduce MAP activity [64]. Taken together, analyses of the effects of the MAP on these drugs and the effects of these drugs on MAP can shed substantial light on the possibility that synergy between the drugs is due to mutual inhibition of their biotransformation either directly or through their metabolites, and that signaling-changes are secondary. Competitive inhibition of either GST or RLIP76 due to metabolites would be a potential reason for increased accumulation of proapoptotic lipid aldehydes in CaP cells.

\section{Role of Ral signaling in the antineoplastic activity of VCN-2 and DTL Cancer generally is} considered as a neoplastic disease with particular causative and etiologic factors as well as protective elements. Although it has remained difficult to treat, it is preventable. Recently, interest in evaluating dietary phytochemical intake as a potential means for chemoprevention of cancer has increased greatly. Regular intake of phytochemicals has been shown to have a cancer protective role during various stages if cancer, including initiation, promotion and progression and, can modulate oncogenic processes through their antioxidant and anti-inflammatory activities of the phytochemicals and their ability to mimic the chemical structure and activity of hormones. The Ral pathway was recently recognized as playing a significant role in malignant transformation, proliferation, invasion, metastasis, and apoptosis pathways in cancers $[28,65,66]$. A role for the Ral pathway in CaP bone metastases is supported by the loss of bone metastatic activity in PC3 cells that have shRNA-mediated decreases in RalA expression [65, 66]. RLIP76 was the first identified, and one of few known effectors of Ral [67-70]. RLIP76 is over- 
expressed in CaP, and inhibition of RLIP76 leads to apoptosis as well as xenograft regression [37]. RLIP76 provides a direct link between the Ras and Ral pathways, linking Ras signaling to metastasis and invasion [28, 67]. Thus, the Ral-RLIP76 interaction could regulate cell motility and invasion through $\mathrm{Ral} / \mathrm{Rac} / \mathrm{cdc} 42$, and cell growth and transformation through Myc. Since Myc regulates p53 [71] and Akt can regulate p53 through MDM2, it is possible that the Ral pathway could regulate Myc and p53 through the effects of RLIP76 on Akt, Rac, or cdc42 [28]. Because Ral signaling has not been explored in CaP, and our studies to date indicate that a Ral effector plays an important role in CaP [37], these findings point to a significant role of Ral signaling in CaP biology (Fig. 3). Further investigation in this area will delineate the mechanisms through which VCN-2 exerts its anticancer effects in $\mathrm{CaP}$ and perhaps the mechanisms of VCN-2/DTL interactions.

\section{AICaP cells are sensitive to VCN-2, and the VCN-2/DTL combination exerts synergy}

Recent studies demonstrated that VCN-2 can potently inhibit the growth of CaP in-vitro as well as invivo, as compared to other active constituents of OSL like orientin and luteolin. VCN-2 increased levels of PTEN, decreased levels of hTERT, PCNA, and pRB, and increased p53 levels while inhibiting pEGFR, pAkt, and the mTOR signaling mediator pP70S6K. VCN-2 induced G2/M arrest by decreasing cyclin B1, cyclin D1, and CDK4, and inhibited angiogenesis both in-vitro and in-vivo, as shown by decreases in VEGF, PSMA, and angiogenic marker CD31. Most importantly, VCN-2 as a single agent decreased the levels of AR, C-Myc, and Bcl2 and inhibited the activation of pIGF-1R, which is of specific significance to chemo-dietary therapy of castration resistant CaP (CRCaP). In-vivo mice xenografts studies in which VCN-2 was administered by oral gavage revealed potent regression of CRCaP xenografts with a synergistic effect with DTL, the current drug of choice in CRCaP [33]. VCN-2 alone or in combination with DTL did not cause any overt toxicity in mice. The rationale is based on the entirely novel concept that the Ral-signaling pathway that signals $\mathrm{CaP}$ growth and metastasis and the mercapturic acid toxinbiotransformation pathway that protects cells from toxins are together responsible for the aggressive behavior of AICaP. Further studies will facilitate the translation of VCN-2 into a non-toxic drug useful for improving the DTL efficacy of AICaP.

In summary, $\mathrm{VCN}-2$ has been identified as an anticancer compound present in an herb long thought to be beneficial for men's health and longevity. That VCN-2 can potentiate the anticancer effects of DTL has direct implications in treatment of AICaP. Additional innovation lies in the ability of VCN-2 to recalibrate cellular oncogenic, tumor suppressor and differentiation signaling cascades to induce multispecific anticancer signaling inputs as revealed by our extensive studies in the context of pathogenesis of 
CaP [33]. Also, at orally active and non-toxic doses, VCN-2 induced synergistic anticancer effects with DTL.

\section{Discussion}

Oxidative stress is a well established as contributing to initiation and progression of CaP [46, 72]. GSH, the predominant soluble physiological thiol in cells, is the primary defense against oxidative stress and its levels are increased as a defense against carcinogenesis. The MAP uses GSH to detoxify exogenous and endogenous carcinogenic toxins through enzymatic formation of conjugates of GSH with oxidant toxins (GS-Ox). GSTs are MAP enzymes that catalyze the formation of GS-Ox. The next step towards the excretion of oxidant toxins is their energy-dependent efflux from cells by energy-dependent transporters. RLIP76 (RALBP1) is the most active of these transporters, the loss of which in knockout mice reduces activity of the MAP by 80\% [28, 31, 34-36]. After efflux from cells, GS-Ox are metabolized to mercapturic acids (MA-Ox) and excreted by the kidneys [23]. Chronic exposure to carcinogens activates the MAP, which is very active in CaP [73]. High levels of carcinogen exposure over-rides the protective effect of this detoxification pathway, resulting in sufficient genotoxicity that cell-growth regulatory signaling proteins are altered and cause unregulated growth of the damaged cells and ultimately resulting in cancer $[74,75]$. In addition, expression of this pathway progressively increases during carcinogenesis, such that its activity in cancer cells far exceeds that in normal cells. Indeed, the cancer cells become dependent on this pathway to survive. This is dramatically evident from studies showing that knockout mice lacking RLIP76 are almost entirely resistant to carcinogenesis upon treatment with potent oxidative chemical carcinogens [36, 54]. The importance of RLIP76 for cancer-cell survival is further evident from multiple studies by our [28, 31-37, 54-58] and other [59-62] laboratories showing that blocking the MAP through RLIP76-targeting causes sustained regression of a wide variety of highly treatment-resistant cancers, including $\mathrm{CaP}$ [31, 32, 37], in xenograft mouse models of human cancers.

The name RLIP76 derives from the original identification of this protein as the first (and one of

only a handful of) effector proteins of the Ral-GTPase signaling pathway [67-69]. Ral is a G-protein that is the master regulator of cell motility, membrane-remodeling and ruffling, endocytosis, and actincytoskeleton remodeling that enables cancer cell invasion, metastasis, and stimulation of angiogenesis [28, 76-78]. The importance of RLIP76 in these processes is evident from studies done by us and others showing that angiogenesis as well as invasion or metastasis is severely impaired when RLIP76 is missing or blocked [28, 36, 54-62]. The endocytosis function and GS-Ox efflux functions of RLIP76 are inextricably linked, indicating that this protein serves as a key nexus that links the functions of the Ral 
pathway with the GSH-linked MAP pathway that mitigates oxidative stress [28, 31, 36]. Endocytosis is a key regulator of ligand-receptor signaling that is critical for cancer-cell growth. In addition, activation of Akt, MAPK, Myc, and other cancer promoting signals is regulated by endocytosis [76-78]. This is evident from the deficient functions of these ligands in RLIP76 knockout mice, which are severely deficient in endocytosis [34-36]. Taken together, these observations suggest that multiple cancersignaling mechanisms are controlled by the MAP through the enzymatic activity of RLIP76, and that blockade of its function could serve as an important and effective anticancer therapy [28, 31, 32].

We developed a computer algorithm to perform in-silico screening of flavonoid antioxidant compounds with structures similar to known GST-inhibitors. This strategy was based on known interaction with the MAP through direct quenching lipid-peroxidation of $\omega-6$ polyunsaturated fatty acids, suppression of P450s (including CYP3A4, which metabolizes DTL), induction of GSTs and other GSHlinked enzymes that, together, are chemical and biochemical mechanisms for quenching oxidative stress [23-25]. The docking algorithm utilized coordinates of the complete crystal structure of GST-P [79], the partial crystal structure of RLIP76 [80-82], the known GSH-binding residues and the known kinetic constants for these enzymes [36]. These analyses identified VCN-2 [51, 52]. Our studies of the anticancer activity of VCN-2 towards CaP cells, showed significant anticancer activity in-vitro as well as in-vivo [33]. VCN-2 potentiated the effect of DTL on AICaP, both in-vitro and in-vivo. The anticancer activity of VCN-2 towards the CaP lines LNCaP and PC3 was superior to the activity of resveratrol, silibinin, or curcumin [83-85]. EGF and IGF receptors were inhibited, which was associated with pleiotropic inhibitory effects on signaling proteins down-stream of these cancer-promoting peptide hormones important for CaP growth and invasion [1-4]. Exosome-mediated signaling is a recently identified cancer promoting mechanism. Exosomes are membrane-lipid derived nanoparticles that promote cancer growth [86, 87]. Our preliminary proteomic analyses showed that VCN-2 depleted exosomal RLIP76.

The basic scientific and mechanistic significance of these findings is broader. Many antioxidant compounds that have anticancer activity towards CaP also suppress the MAP pathway and inhibit the enzymatic activity as well as expression of GSTs $[88,89]$. Because the MAP is directly linked to Ral, it is possible that the anticancer effects of several plant-derived antioxidants towards CaP could operate in a similar manner. The structural similarity of the GSH-binding site of GST and RLIP76 supports the idea that they could block MAP by simultaneously inhibiting both enzymes. The observed synergy between DTL and VCN-2 could also be explained by this model because the mechanism of action of DTL is 
through targeting the functions of the cellular cytoskeleton (Fig. 2). Because DTL does not directly affect the MAP, the combination of DTL and VCN-2 could have significant advantages, namely that the functional target of both agents, membrane and cytoskeletal function, would be inhibited through distinct routes such that overlapping toxicities could be avoided. This is supported by the lack of apparent systemic toxicity of the combination of VCN-2 and DTL in mice [33].

VCN-2 exerts highly effective and multi modal regulatory effects on critical nodes of CaP signaling while also exerting a potent synergistic effect with DTL. In this review, we have laid specific emphasis on the critical factors contributing to both primary chemopreventive potential of VCN-2 as well as signaling and metabolic changes that contribute to VCN-2 and DTL synergy, which is relevant for tertiary chemoprevention of even advanced metastatic prostate tumors. We recently showed the efficacy of VCN-2 as a single agent and in combination with DTL in CaP. VCN-2 effectively induced antiproliferative, anti-angiogenic and pro-apoptotic effects in CaP cells (PC-3, DU-145 and LNCaP) regardless of their androgen responsiveness or p53 status. VCN-2 inhibited the EGFR/Akt/mTOR/ p70S6K pathway while decreasing c-Myc, cyclin D1, cyclin B1, CDK4, PCNA and hTERT in-vitro. The potent anticancer effects of VCN-2 seen in in-vitro studies were confirmed in in-vivo nude mice xenograft studies. VCN-2 reached a level of $2.6 \pm 0.3 \mu \mathrm{mol} / \mathrm{L}$ in serum after oral administration in mice, which reflected that VCN-2 is absorbed after oral administration. The i.v. administration of DTL is associated with dose-limiting toxicities such as febrile neutropenia, which has led to characterization of alternate routes of administration and potential combinatorial regimens. In this regard, in a preclinical model, VCN-2 in combination with DTL synergistically inhibited the growth of prostate tumors in-vivo with a greater decrease in the levels of AR, pIGF1R, pAkt, PCNA, cyclin D1, Ki67, CD31, and increase in Ecadherin [33]. VCN-2 has also been investigated for radioprotection and anti-inflammatory properties [51, 52]. These findings collectively provide strong evidence that VCN-2 is effective against CaP progression along with indicating that VCN-2 and DTL co-administration is more effective than either of the single agents in AICaP. Therefore, we believe that chronic administration of VCN-2 and/or its chemical derivatives have strong potential for use as preventive agents for $\mathrm{CaP}$, and that VCN-2 could be used to enhance the anticancer efficacy of chemotherapy drugs. High intensity acute exposure to electrophilic compounds (i.e., chemotherapy drugs) causes apoptosis and necrosis; chronic long-term exposure accelerates carcinogenesis. The endogenous toxin metabolism is primarily responsible for biotransformation of lipid-hydroperoxides $(\mathrm{LOOH})$ and their degradation products into mercapturic acids (Fig. 3). LOOH decompose into toxic electrophilic aldehydes that are pro-apoptotic and mutagenic alkylating agents [23]. Currently, the clinical use of DTL is associated with need for higher doses in 
elderly patients, who form the largest group of individuals affected with CRCaP, leading to substantial toxicity [13]. The pharmacokinetic studies will provide requisite rationale and dosage calibrations that are essential for further studies. Spontaneous tumor models represent the best means by which to test the primary chemo-preventative effect of VCN-2 and in which to establish a reliable set of VCN-2 regulated secretory biomarkers.

\section{Conclusion}

Regression of AICaP cell line PC3 upon RLIP76 blockade, inhibition of growth of PC3 by a novel plant polyphenol, VCN-2, and the synergy between VCN-2 and DTL in xenografts are novel findings [33, 37]. The interactions between Ral and the MAP are novel and the potential role of these in regulation of exosome signaling could represent a paradigm shift in CaP biology. VCN-2 causes apoptosis in ASCaP as well as in AICaP. It simultaneously inhibits multiple signals downstream of EGF and IGF that are important for the aggressive behavior of AICaP. These include signals that regulate cell cycle check points (p53, Rb, PCNA), proliferation (PCNA), differentiation (cМyc, E-cadherin, fibronectin), survival (Akt, mTOR), senescence (hTERT), and apoptosis (bcl-2, Bax, Bak, caspases) [33]. In ASCaP, these signaling changes are accompanied by down-regulation of AR. The underlying mechanisms of the wide spectrum of anticancer signaling effects are incompletely understood. Studies by Nagaprashantha et al. indicate that VCN-2 depletes RLIP76 in exosomes secreted by CaP. These CaP-secreted exosomes function in an autocrine manner to activate the Ral-A pathway, which controls the movement and remodeling of membranes and cytoskeleton, and serves as a generalized modulator of ligand-receptor signaling by regulating endocytosis [86, 87]. Because DTL also targets the cytoskeleton, we postulate that the mechanism of action of VCN-2, the synergy between DTL and VCN-2, and pleiotropic signaling effects occur through the effects of VCN-2 on Ral, a pathway that is regulated by MAP. Recent studies revealed the effect of VCN-2 on signaling networks of Ral and their relationship to proteins that serve to promote AICaP growth. These studies addressed generalizability and mechanisms in-vitro and confirmed in animal xenograft studies that should simultaneously examine potential pharmacokinetic and toxicological interaction of VCN-2 and DTL combination.

The overall objective of our proposed combinatorial (VCN2 + DTL) approach is to improve the length and quality of life of men with metastatic AICaP by increasing the effectiveness of DTL chemotherapy by using the non-toxic and active natural compound VCN-2. Our immediate future objectives are to understand whether these observations are broadly applicable to AICaP in cell lines, to define the mechanism of action of VCN-2, and to determine the mechanism underlying its anticancer 
synergy with DTL. These studies will provide a much needed understanding of how AICaP cells survive and grow, and could lead to additional treatment approaches. This review has summarized our current understanding of VCN-2 is a potent, natural anticancer compound that broadly targets MAP and causes cancer-selective apoptosis that can be used to prevent and treat $\mathrm{CaP}$, and that the effects of VCN-2 can be monitored through the CaP-specific exosomal fraction in peripheral blood. Therefore, the present preclinical findings of detailed cellular, genetic and pharmacokinetic mechanisms of action would lay strong scientific foundation for further clinical development of VCN-2 chemo-dietary regimens for effective management of human CaP. Future studies are needed to advance scientific knowledge in the role of Ralsignaling in CaP biology, define this novel mechanism of action of antioxidant antineoplastic agents, and allow development of new strategies to defeat DTL-resistance in AICaP.

\section{Relevance to Human Health}

VCN-2 is a safe flavonoid that is enriched in the time-tested Indian herb Tulsi, represents a potent and effective chemopreventive compound whose multi-specific anticancer signaling properties are highly relevant to the extensive genetic and molecular complexity associated with the primary pathogenesis of $\mathrm{CaP}$, as well as the emergence of AICaP. VCN-2 has potent anticancer activity in both ASCaP and AICaP, and remarkable synergy when used with DTL in cell and human xenograft models. VCN-2 effectively and simultaneously targets many essential molecular networks that influence CaP incidence, acquisition and maintenance of pluripotency, tumor angiogenesis, apoptosis resistance, and development of AICaP and metastasis. Our future studies aim to delineate the mechanistic basis underlying the usefulness of VCN-2 alone for chemoprevention of CaP and AICaP, as well as to evaluate the combination of VCN-2 and DTL as a tertiary chemo-dietary approach to prevent aggressive and metastatic CaP. In this regard, determining the efficacy of VCN-2 in pre-clinical models of CaP could have significant impact by informing the rational use of VCN-2 as well as VCN-2 and DTL combinatorial regimens in human CaP prevention and control.

\section{Future Perspectives}

The use of VCN-2 as an anti-prostate cancer natural agent is novel. The potent interaction of VCN-2 with DTL is novel and of immediate significance in the current therapy of AICaP. The prediction algorithm for anticancer activity of antioxidants based on in-silico models of interaction between detoxification enzymes and signaling proteins is novel, and appears to be highly significant based on correct predictions of the activity of VCN-2 in CaP. The results described herein have provided strong preclinical rationale for the anticancer effects of VCN-2 in CaP by revealing the mechanisms of regulation of critical nodes of 
CaP such as cMyc, AR, and p53. The systematic investigation of secretory and exosomes proteins invitro along with analysis of differentially detected proteins in in-vivo spontaneous models of control and VCN-2 treated groups will help to establish a reliable set of VCN-2 response predictive, CaP-specific biomarkers for further studies. The spontaneous models will also help to strengthen the requisite preclinical rationale to pursue clinical trials. Collectively, these studies will bring about a paradigm shift in the field of $\mathrm{CaP}$ research by laying greater emphasis on developing the non-toxic flavonoid $\mathrm{VCN}-2$ for effective interventional strategies for CaP. 
Acknowledgments This work was supported in part by the Department of Defense grant (W81XWH-16-1-0641) and funds from the Perricone Family Foundation, Los Angeles, CA. Funding from the Beckman Research Institute of City of Hope is also acknowledged. We apologize to all colleagues whose work we could not cite due to space constraints. 


\section{References}

1 G. Attard, C. Parker, R.A. Eeles, F. Schröder, S.A. Tomlins, I. Tannock, C.G. Drake, J.S. de Bono. Prostate cancer. Lancet. 2016; 387: 70-82.

2 A.J. Chang, K.A. Autio, M. Roach, H.I. Scher. High-risk prostate cancer-classification and therapy. Nat Rev Clin Oncol. 2014; 11: 308-323.

3 W.G. Nelson, A.M. De Marzo, W.B. Isaacs. Prostate cancer. The New Eng J Med. 2003; 349: 366-381.

4 A.M. Aschelter, S. Giacinti, P. Caporello, P. Marchetti. Genomic and epigenomic alterations in prostate cancer. Front Endocrinol. 2012; 3: 128.

5 M.M. Shen, C. Abate-Shen. Molecular genetics of prostate cancer: new prospects for old challenges. Genes Dev. 2010; 24(18): 1967-2000.

6 J.T. Arnold, J.T. Isaacs. Mechanisms involved in the progression of androgen-independent prostate cancers: it is not only the cancer cell's fault. Endocr Relat Cancer. 2002; 9(1): 61-73.

7 B.J. Feldman, D. Feldman. The development of androgen-independent prostate cancer. Nature Rev Cancer. 2001; 1: 34-45.

8 K.J. Pienta, D. Bradley. Mechanisms underlying the development of androgen-independent prostate cancer. Clin Cancer Res. 2006; 12(6): 1665-1671.

$9 \quad$ S.A. Tomlins, R. Mehra, D.R. Rhodes, X. Cao, L. Wang, S.M. Dhanasekaran, S. KalyanaSundaram, J.T. Wei, M.A. Rubin, K.J. Pienta, R.B. Shah, A.M. Chinnaiyan. Integrative molecular concept modeling of prostate cancer progression. Nat Genet. 2007; 39: 41-51.

10 M. Andreoiu, L. Cheng. Multifocal prostate cancer: biologic, prognostic, and therapeutic I mplications. Hum Pathol. 2010; 41: 781-793.

11 S.E. Delacroix, J.F. Ward. Prostate cancer multifocality: impact on cancer biology and treatment recommendations. Panminerva Med. 2010; 52: 209-216.

12 M.C. Lee, A.S. Moussa, C. Yu, M.W. Kattan, C. Magi-Galluzzi, J.S. Jones. Multifocal high grade prostatic intraepithelial neoplasia is a risk factor for subsequent prostate cancer. J Urol. 2010; 184: 1958-1962.

13 F.K. Engels, J. Verweij. Docetaxel administration schedule: from fever to tears? A review of randomised studies. Eur J Cancer. 2005; 41: 1117-1126.

14 H. Kenmotsu, Y. Tanigawara. Pharmacokinetics, dynamics and toxicity of docetaxel: Why the Japanese dose differs from the Western dose. Cancer Sci. 2015; 106(5): 497-504. 
15 H.F. Ji, X.J. Li, H.Y. Zhang. Natural products and drug discovery. Can thousands of years of ancient medical knowledge lead us to new and powerful drug combinations in the fight against cancer and dementia? EMBO Rep. 2009; 10(3): 194-200.

16 G.M. Cragg, D.J. Newman. Natural products: a continuing source of novel drug leads. Biochim Biophys Acta. 2013; 1830(6): 3670-3695.

17 A.A. Siddiqui, F. Iram, S. Siddiqui, K. Sahu. Role of natural products in drug discovery process. Int J Drug Dev Res. 2014; 6(2): 172-204.

18 H. Wang, T.O. Khor, L. Shu, Z. Su, F. Fuentes, J.H. Lee, A.N. Tony Kong. Plants against cancer: a review on natural phytochemicals in preventing and treating cancers and their druggability. Anticancer Agents Med Chem. 2012; 12(10): 1281-1305.

19 A. Niedzwiecki, M.W. Roomi, T. Kalinovsky, M. Rath. Anticancer efficacy of polyphenols and their combinations. Nutrients 2016; 8: 552.

20 S. Elmore. Apoptosis: a review of programmed cell death. Toxicol Pathol. 2007; 35(4): 495-516.

21 C. Wang, R.J. Youle. The role of mitochondria in apoptosis. Annu Rev Genet. 2009; 43: 95-118.

22 D. Xiao, K.L. Lew, Y.A. Kim, Y. Zeng, E.R. Hahm, R. Dhir, S.V. Singh. Diallyl trisulfide suppresses growth of PC-3 human prostate cancer xenograft in vivo in association with Bax and Bak induction. Clin Cancer Res. 2006; 12: 6836-6843.

23 W.B. Jakoby. The glutathione S-transferases: a group of multi-functional detoxification protein. Adv Enzymol Mol Biol. 1978; 46: 383-414.

24 Y.C. Awasthi, R. Sharma, S.S. Singhal. Human Glutathione S-transferases. Int J Biochem. 1994; 26: 295-308.

25 J.D. Hayes, D.J. Pulford. The glutathione S-transferase supergene family: regulation of GST and the contribution of the isoenzymes to cancer chemoprotection and drug resistance. Crit Rev Biochem Mol Biol. 1995; 30: 445-600.

26 T. Matsuzaki, S. Hanai, H. Kishi, Z. Liu, Y. Bao, A. Kikuchi, K. Tsuchida, H. Sugino. Regulation of endocytosis of activin type II receptors by a novel PDZ protein through Ral/Ral-binding protein 1-dependent pathway. J Biol Chem. 2002; 277: 19008-19018.

27 J.J. Gildea, M.A. Harding, M.J. Seraj, K.M. Gulding, D. Theodorescu. The role of Ral A in epidermal growth factor receptor-regulated cell motility. Cancer Res. 2002; 62: 982-985.

28 S. Awasthi, S.S. Singhal, R. Sharma, P. Zimniak, Y.C. Awasthi. Transport of glutathioneconjugates and chemotherapeutic drugs by RLIP76: a novel link between G-protein and tyrosinekinase signaling and drug-resistance. Int J Cancer. 2003; 106: 635-646. 
F. Chang, L.S. Steelman, J.T. Lee, J.G. Shelton, P.M. Navolanic, W.L. Blalock, R.A. Franklin, J.A. McCubrey. Signal transduction mediated by the Ras/Raf/MEK/ERK pathway from cytokine receptors to transcription factors: potential targeting for therapeutic intervention. Leukemia. 2003; 17: $1263-1293$.

30 C.V. Dang. MYC on the path to cancer. Cell. 2012; 149(1): 22-35.

31 S. Awasthi, S.S. Singhal, Y.C. Awasthi, B. Martin, J.H. Woo, C.C. Cunningham, A.E. Frankel. RLIP76 and cancer. Clin Cancer Res. 2008; 14: 4372-4377.

32 S.S. Singhal, S. Yadav, C. Roth, J. Singhal. RLIP76: A novel glutathione-conjugate and multidrug transporter. Biochem Pharmacol. 2009; 77: 761-769.

33 L.D. Nagaprashantha, R. Vatsyayan, J. Singhal, S. Fast, R. Roby, S. Awasthi, S.S. Singhal. Anticancer effects of novel flavonoid vicenin-2 as a single agent and in synergistic combination with docetaxel in prostate cancer. Biochem Pharmacol. 2011; 82: 1100-1109.

34 S. Awasthi, S.S. Singhal, S. Yadav, J. Singhal, K. Drake, A. Nadkar, E. Zajac, D. Wickramarachchi, N. Rowe, A. Yacoub, P. Boor, S. Dwivedi, P. Dent, Y.C. Awasthi. RALBP1 is a major determinant of radiation sensitivity. Cancer Res. 2005; 65: 6022-6028.

35 J. Singhal, S.S. Singhal, S. Yadav, M. Warnke, A. Yacoub, P. Dent, R. Sharma, Y.C. Awasthi, D. Armstrong, S. Awasthi. RLIP76 in defense of radiation poisoning. Int J Rad Oncol Biol Phys. 2008; 72: 553-561.

36 S.S. Singhal, D. Wickramarachchi, S. Yadav, J. Singhal, K. Leake, R. Vatsyayan, P. Lelsani, P. Chaudhary, S. Suzuki, S. Yang, Y.C. Awasthi, S. Awasthi. Glutathione-conjugate transport by RLIP76 is required for clathrin-dependent endocytosis and chemical carcinogenesis. Mol Cancer Therap. 2011; 10: 16-28.

37 S.S. Singhal, C. Roth, K. Leake, J. Singhal, S. Yadav, S. Awasthi. Regression of prostate cancer xenografts by RLIP76 depletion. Biochem Pharmacol. 2009; 77: 1074-1083.

38 S. Singh, D.K. Majumdar, H.M. Rehan. Evaluation of anti-inflammatory potential of fixed oil of ocimum sanctum (holybasil) and its possible mechanism of action. J Ethnopharmacol. 1996; 54: 19-26.

39 V. Rai, U. Iyer, U.V. Mani. Effect of tulasi (ocimum sanctum) leaf powder supplementation on blood sugar levels, serum lipids and tissue lipids in diabetic rats. Plant Foods Hum Nutr. 1997; 50: 9-16.

40 C. Marrassini, R. Davicino, C. Acevedo, C. Anesini, S. Gorzalczany, G. Ferraro. Vicenin-2, a potential anti-inflammatory constituent of urtica circularis. J Nat Prod. 2011; 74 (6): 1503-1507. 
41 M. Satyamitra, S. Mantena, C.K.K. Nair, S. Chandna, B.S. Dwarakanath, P. Uma Devi. The antioxidant flavonoids, orientin and vicenin enhance repair of radiation-induced damage. SAJ Pharma Pharmacol. 2014; 1: 105.

42 M.N. Islam, I.J. Ishita, H.A. Jung, J.S. Choi. Vicenin-2 isolated from Artemisia capillaris exhibited potent anti-glycation properties. Food Chem Toxicol. 2014; 69: 55-62.

43 I.C. Lee, J.S. Bae. Anti-inflammatory effects of vicenin-2 and scolymoside on polyphosphatemediated vascular inflammatory responses. Inflamm Res. 2016; 65: 203-212.

44 P. Dharmani, V.K. Kuchibhotla, R. Maurya, S. Srivastava, S. Sharma, G. Palit. Evaluation of anti-ulcerogenic and ulcer-healing properties of Ocimum sanctum Linn. J Ethnopharmacol. 2004; 93: 197-206.

45 V. Vats, S.P. Yadav, J.K. Grover. Ethanolic extract of Ocimum sanctum leaves partially attenuates streptozotocin-induced alterations in glycogen content and carbohydrate metabolism in rats. J Ethnopharmacol. 2004; 90: 155-160.

46 M.A. Trush, T.W. Kensler. An overview of the relationship between oxidative stress and chemical carcinogenesis. Free Radic Biol Med. 1991; 10: 201-209.

47 A.L. Sabichi, S.M. Lippman. COX-2 inhibitors and other nonsteroidal anti-inflammatory drugs in genitourinary cancer. Semin Oncol. 2004; 31: 36-44.

48 R.P. Singh, G. Sharma, S. Dhanalakshmi, C. Agarwal, R. Agarwal. Suppression of advanced human prostate tumor growth in athymic mice by silibinin feeding is associated with reduced cell proliferation, increased apoptosis, and inhibition of angiogenesis. Cancer Epidemiol Biomarkers Prev. 2003; 12: 933-939.

49 A.I. Amjad, R.A. Parikh, L.J. Appleman, E.R. Hahm, K. Singh, S.V. Singh. Broccoli-derived sulforaphane and chemoprevention of prostate cancer: from bench to bedside. Curr Pharmacol Rep. 2015; 1(6): 382-390.

50 K. Karthikeyan, P. Ravichandran, S. Govindasamy. Chemopreventive effect of ocimum sanctum on DMBA-induced hamster buccal pouch carcinogenesis. Oral Oncol. 1999; 35: 112-119.

51 V. Nayak, P.U. Devi. Protection of mouse bone marrow against radiation-induced chromosome damage and stem cell death by the ocimum flavonoids orientin and vicenin. Radiat Res. 2005; 163:165-171.

52 B. Vrinda, P.U. Devi. Radiation protection of human lymphocyte chromosomes in vitro by orientin and vicenin. Mutat Res. 2001; 498; 39-46.

53 S. Mondal, B.R. Mirdha, S.C. Mahapatra. The science behind sacredness of Tulsi (Ocimum sanctum Linn.). Ind J Physiol Pharmacol. 2009; 53 (4): 291-306. 
54 S. Lee, J. Wurtzel, S.S. Singhal, S. Awasthi, L.E. Goldfinger. RALBP1/RLIP76 depletion in mice suppresses tumor growth by inhibiting tumor neo-vascularization. Cancer Res. 2012; 72: 5165-5173.

55 S.S. Singhal, J. Singhal, S. Yadav, M. Sahu, Y.C. Awasthi, S. Awasthi. RLIP76: a target for kidney cancer therapy. Cancer Res. 2009; 69: 4244-4251.

56 S.S. Singhal, J. Singhal, S. Yadav, S. Dwivedi, P. Boor, S. Awasthi. Regression of lung and colon cancer xenografts by depleting or inhibiting RLIP76 (RALBP1). Cancer Res. 2007; 67: 4382-4389.

57 K. Leake, J. Singhal, L. Nagaprashantha, S. Awasthi, S.S. Singhal. RLIP76 regulates PI3K/Akt signaling and chemo-radio-therapy resistance in pancreatic cancer. PLoS ONE. 2012; 7, e34582.

58 S.S. Singhal, Y.C. Awasthi, S. Awasthi. Regression of melanoma in a murine model by RLIP76 depletion. Cancer Res. 2006; 66: 2354-2360.

59 Q. Wang, J.Y. Wang, X.P. Zhang, Z.W. Lv, D. Fu, Y.C. Lu, G.H. Hu, C. Luo, J.X. Chen. RLIP76 is overexpressed in human glioblastomas and is required for proliferation, tumorigenesis and suppression of apoptosis. Carcinogenesis. 2013; 34: 916-926.

60 Z. Wu, C. Owens, N. Chandra, K. Popovic, M. Conaway, D. Theodorescu. RalBP1 is necessary for metastasis of human cancer cell lines. Neoplasia. 2010; 12: 1003-1012.

61 T.D. Martin, J.C. Samuel, E.D. Routh, C.Y. Der, J.J. Yeh. Activation and involvement of Ral GTPases in colorectal cancer. Cancer Res. 2011; 71,: 206-215.

62 Q. Wang, J. Qian, J.Y. Wang, C. Luo, J. Chen, G. Hu, Y. Lu. Knockdown of RLIP76 expression by RNA interference inhibits invasion, induces cell cycle arrest, and increases chemosensitivity to the anticancer drug temozolomide in glioma cells. J Neurooncol. 2013; 112: 73-82.

63 R. Václavíková, S. Horský, P. Simek,I. Gut. Paclitaxel metabolism in rat and human liver microsomes is inhibited by phenolic antioxidants. Naunyn Schmiedebergs Arch Pharmacol. 2003; 368: 200-209.

64 S. Awasthi, S.K. Srivastava, F. Ahmad, H. Ahmad, G.A.S. Ansari. Interactions of glutathione Stransferase $\pi$ with ethacrynic acid and its glutathione conjugate. Biochim Biophys Acta. 1993; 1164: 173-178.

65 A.A. Adjei. Blocking oncogenic Ras signaling for cancer therapy. J Natl Cancer Inst. 2001; 93: 1062-1074.

66 P.J. Roberts, C.J. Der. Targeting the Raf-MEK-ERK mitogen-activated protein kinase cascade for the treatment of cancer. Oncogene. 2007; 26: 3291-3310. 
67 V. Jullien-Flores, F. Dorseuil O, Romero, F. Letourneur, S. Saragosti, R. Berger, A. Tavitian, G. Gacon, J.H. Camonis. Bridging Ral GTPase to Rho pathways. RLIP76, a Ral effector with CDC42/Rac GTPase-activating protein activity. J Biol Chem. 1995; 270: 22473-22477.

68 S.H. Park, R.A. Weinberg. A putative effector of Ral has homology to Rho/Rac GTPase activating proteins. Oncogene. 1995; 11: 2349-2355.

69 S.B. Cantor, T. Urano, L.A. Feig. Identification and characterization of Ral-binding protein 1, a potential downstream target of Ral GTPases. Mol Cell Biol. 1995; 15: 4578-4584.

70 S. Moskalenko, D.O. Henry, C. Rosse, G. Mirey, J.H. Camonis, M.A. White. The exocyst is a Ral effector complex. Nat Cell Biol. 2002; 4: 66-72.

71 M. Sachdeva, S. Zhu, F. Wu, H. Wu, V. Walia, S. Kumar, R. Elble, K. Watabe, Y.Y. Mo. p53 represses c-Myc through induction of the tumor suppressor miR-145. Proc Natl Acad Sci. 2009; 106: 3207-3212.

72 C.P. Nelson, L.C. Kidd, J. Sauvageot, W.B. Isaacs, A.M. De Marzo, J.D. Groopman, W.G. Nelson, T.W. Kensler. Protection against 2-hydroxyamino-1-methyl-6-phenylimidazo[4,5b]pyridine cytotoxicity and DNA adduct formation in human prostate by glutathione Stransferase P1. Cancer Res. 2001; 61: 103-109.

73 A.M. De Marzo, E.A. Platz, S. Sutcliffe, J. Xu, H. Grönberg, C.G. Drake, Y. Nakai, W.B. Isaacs, W.G. Nelson. Inflammation in prostate carcinogenesis. Nat Rev Cancer. 2007; 7(4): 256-269.

74 D. Hanahan, R.A. Weinberg. The hallmarks of cancer. Cell. 2000; 100: 57-70.

75 C.L. Chaffer, R.A. Weinberg. A perspective on cancer cell metastasis. Science. 2011; 331: 15591564.

76 C. Rosse, S. L'Hoste, N. Offner, A. Picard, J.H. Camonis. RLIP, an effector of the Ral-GTPases, is a platform for Cdk1 to phosphorylate epsin during the switch off of endocytosis in mitosis. $\mathrm{J}$ Biol Chem. 2003; 278: 30597-30604.

77 K. Morinaka, S. Koyama, S. Nakashima,T. Hinoi, K. Okawa, A. Iwamatsu, A. Kikuchi. Epsin binds to the EH domain of POB1 and regulates receptor-mediated endocytosis. Oncogene.1999; 18: 5915-5922.

78 V. Jullien-Flores, Y. Mahe, G. Mirey, C. Leprince, B. Meunier-Bisceuil, A. Sorkin, J.H. Camonis. RLIP76, an effector of the GTPase Ral, interacts with the AP2 complex: involvement of the Ral pathway in receptor-endocytosis. J Cell Sci. 2000; 113: 2837-2844.

79 T. Kano, M. Sakai, M. Muramatsu. Structure and expression of a human class pi glutathione Stransferase messenger RNA. Cancer Res . 1987; 47: 5626-5630. 
80 H.R. Mott, D. Owen. RLIP76 (RalBP1): The first piece of the structural puzzle. Small GTPases. 2010; 1: 157-160.

81 K.V. Rajasekar, L.J. Campbell, D. Nietlispach, D. Owen, H.R. Mott. The structure of the RLIP76 RhoGAP-Ral binding domain dyad: fixed position of the domains leads to dual engagement of small G proteins at the membrane. Structure. 2013; 21: 2131-2142.

82 H.R. Mott, D. Owen. Structure and function of RLIP76 (RalBP1): an intersection point between Ras and Rho signalling. Biochem Soc Trans. 2014; 42: 52-58.

83 K.J. Wu, J. Zeng, G.D. Zhu, L.L. Zhang, D. Zhang, L. Li, J.H. Fan, X.Y. Wang, D.L. He. Silibinin inhibits prostate cancer invasion, motility and migration by suppressing vimentin and MMP-2 expression. Acta Pharmacol Sin. 2009; 30: 1162-1168.

84 R.P. Singh, G. Deep, M.J. Blouin, M.N. Pollak, R. Agarwal. Silibinin suppresses in vivo growth of human prostate carcinoma PC-3 tumor xenograft. Carcinogenesis. 2007; 28: 2567-2574.

85 M. Li, Z. Zhang, D.L. Hill , H. Wang, R. Zhang. Curcumin, a dietary component, has anticancer, chemo-sensitization, and radio-sensitization effects by down-regulating the MDM2 oncogene through the PI3K/mTOR/ETS2 pathway. Cancer Res. 2007; 67: 1988-1996.

86 C. Villarroya-Beltria, F. Baixaulia, C. Gutiérrez-Vázqueza, F. Sánchez-Madrida, M. Mittelbrunna. Sorting it out: Regulation of exosome loading. Seminars Cancer Bio. 2014; 28: 313.

87 Y. Sun, J. Liu. Potential of cancer cell-derived exosomes in clinical application: a review of recent research advances. Clin Therap. 2014; 36: 863-872.

88 S. Cheng, N. Gao, Z. Zhang, G. Chen, A. Budhraja, Z. Ke, Y.O. Son, X. Wang, J. Luo, X. Shi. Quercetin induces tumor-selective apoptosis through downregulation of Mcl-1 and activation of Bax. Clin Cancer Res. 2010; 16: 5679-5691.

89 N.M. Meybodi, A.M. Mortazavian, A.B. Monfared, S. Sohrabvandi, F.A. Meybodi. Phytochemicals in cancer prevention: a review of the evidence. Iran J Cancer Prev. 2017; e7219. 


\section{Figure Legends}

Figure 1: Chemical structure of vicenin-2 (VCN-2)

Figure 2: Mechanism of synergistic action of VCN-2 and docetaxel (DTL)

Figure 3: Ral-MAP pathway interactions Stress causes activation of Ral to Ral GTP and the formation of $\mathrm{LOOH}$ from membrane lipids. This causes dissociation of the RLIP76

(RalBP1)/Hsf1/Hsp90/Tubulin complex, releasing Hsf1 for translocation to the nucleus in a complex with p53. RLIP76 translocates to the cell membrane, where it binds to clathrin adaptors and links its ATPase coupled GS-HNE transport with clathrin-dependent endocytosis. EGF-EGFR or IGF-IGFR complex is endocytosed and, subsequently, downstream kinase regulation is activated leading to p53 and cMyc regulation. $\mathrm{LOOH}$ is metabolized to $4 \mathrm{HNE}$ by p450, then to GS-HNE by GST. GS-HNE is transported out by RLIP76. 


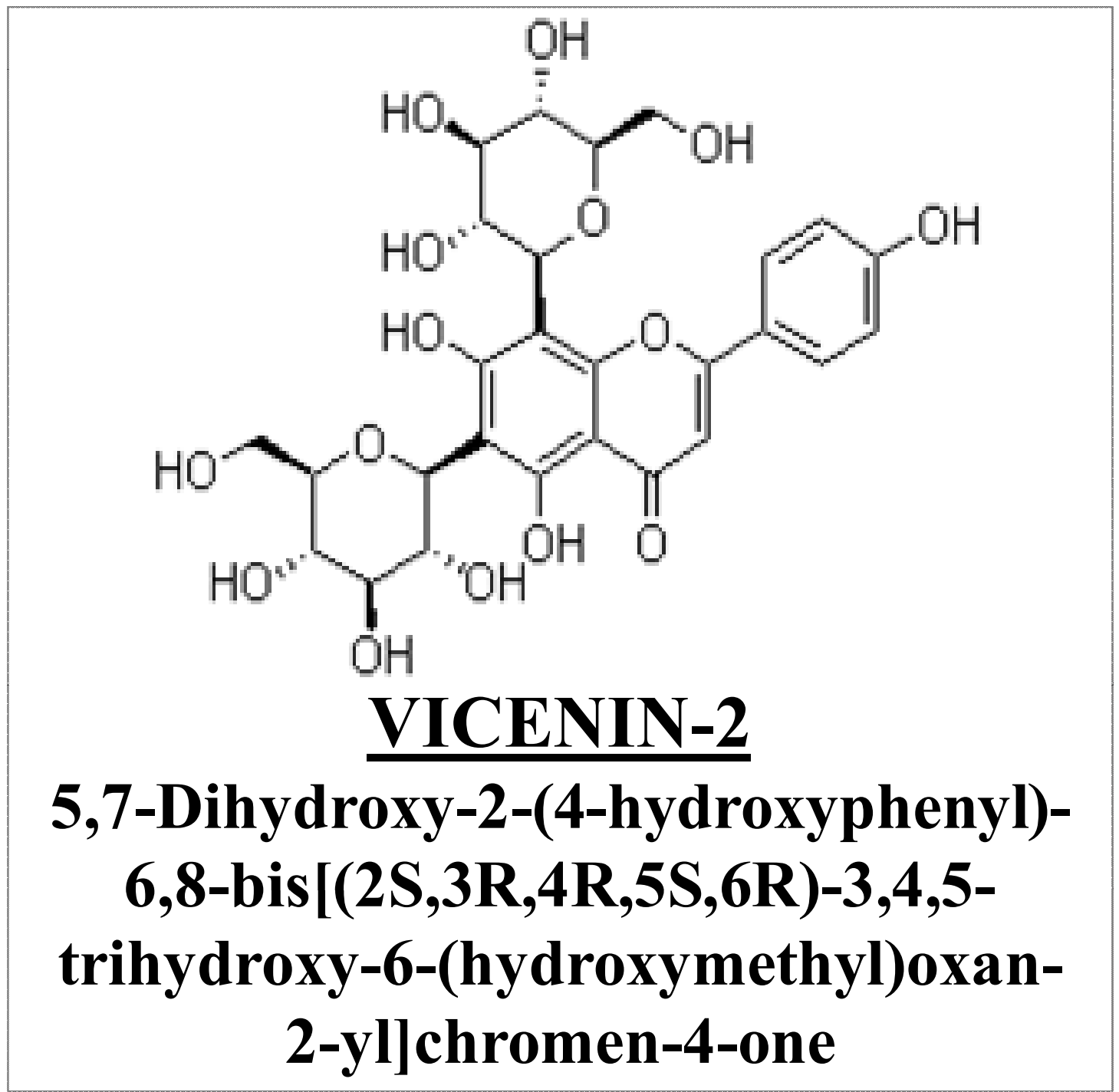




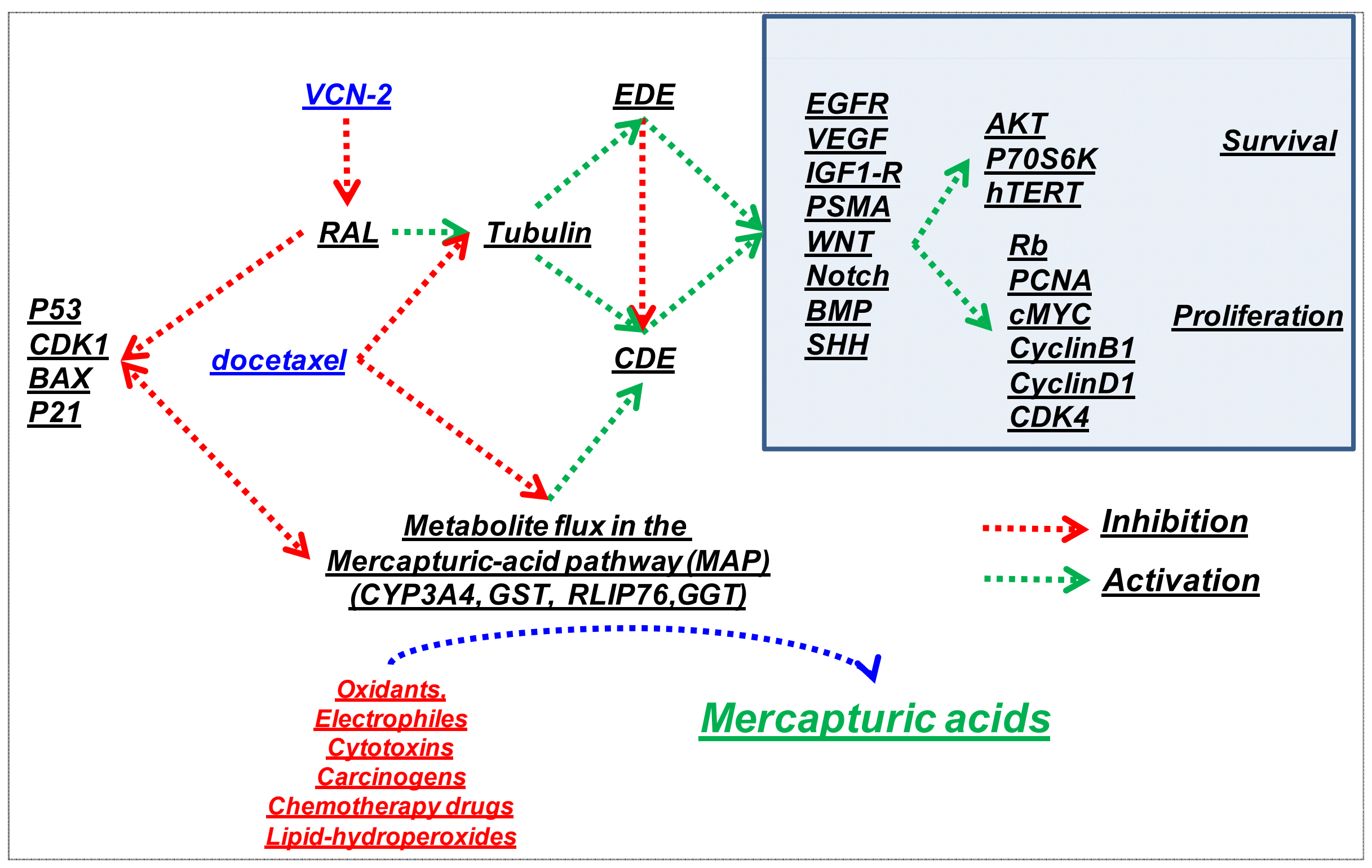


Singhal et al., Figure 3

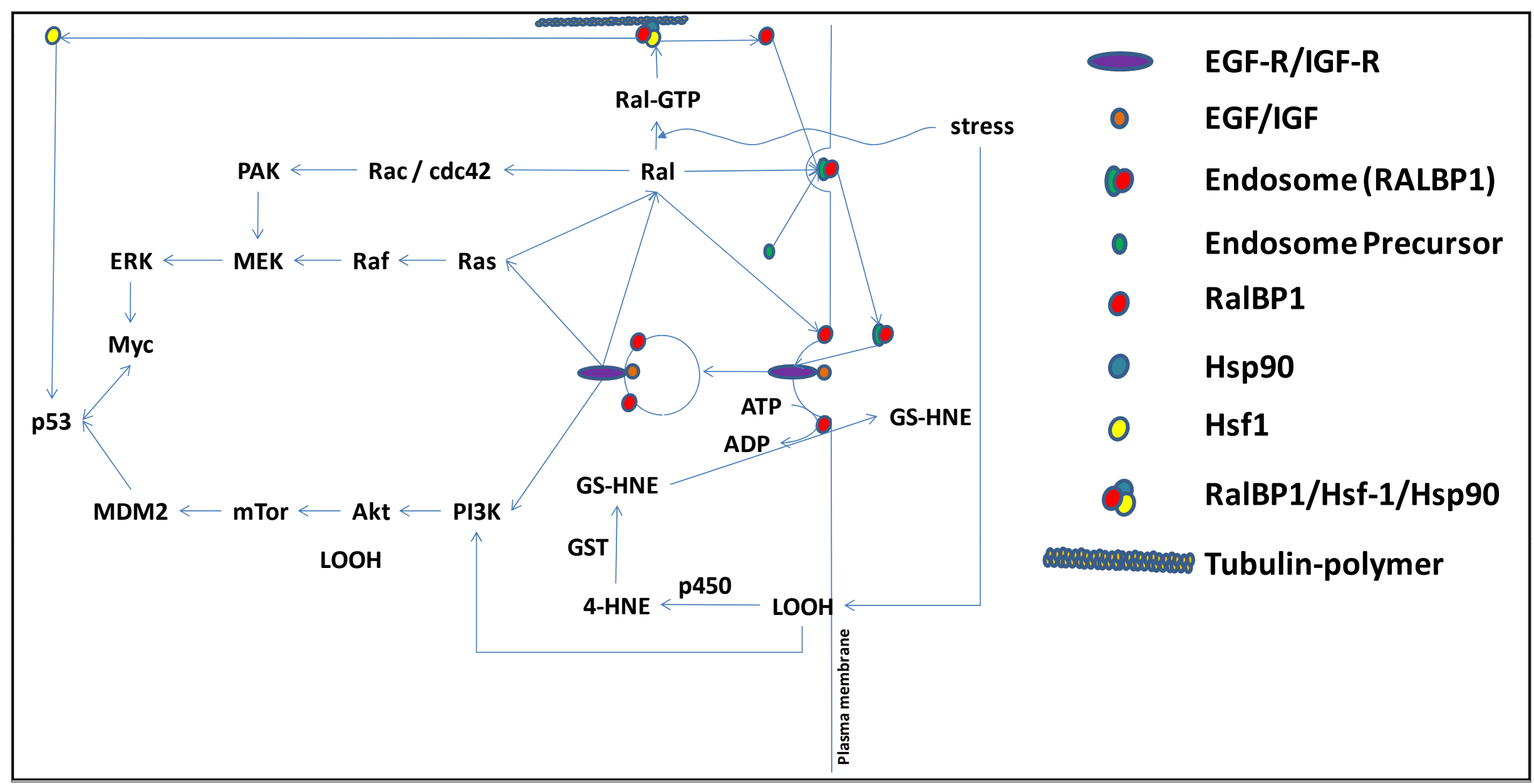

